\title{
Concentration-reinfusion (CR) of ascitic fluid in the treatment of decompensated liver cirrhosis with diuretic-resistant ascites
}

\author{
E. VENTURA \\ M.D.
}

\author{
M. L. ZENEROLI \\ M.D.
}

Institute of Clinical Medicine, University of Modena, Italy

\begin{abstract}
Summary
The ascites concentration-reinfusion as current therapeutic procedure is probably too expensive (but less expensive than desalinated human albumin) and in our opinion it is not a substitute for the diuretic drugs in diuretic-responsive ascites.

On the other hand, this method plays an important role in the management of diuretic-resistant ascites. The mechanical extraction of the sequestered fluid and the infusion of a concentrated derivative into the blood stream beyond the portal blockade aim to restore the 'effective' blood volume, renal perfusion and GFR. This effect may induce a new responsive state of the kidney to diuretic drugs and may correct the functional renal failure.
\end{abstract}

In addition, a well concentrated ascites supplies autologous proteins to those patients with gross abnormalities of serum protein concentrations.

THE rational management of ascites in liver cirrhosis is by sodium restricted diet and drugs, inducing water and sodium excretion by different and complementary mechanisms.

A wide clinical experience has shown that the best therapeutic effects are obtained by means of drugs acting on the distal tubule of the nephron (spironolactone, triamterene, amiloride), possibly associated with diuretics acting on proximal tubule and loop of Henle (ethacrynic acid and frusemide).

However, the progressive fall of the serum albumin concentration and the haemodynamic imbalance in the portal and splanchnic circulation (mainly the lymph drainage) again induces ascites formation. Hence, the shifting of fluid from systemic vascular bed to peritoneal space decreases the 'effective' blood volume and renal perfusion and enhances the hormonal factors involved in the abnormal water and sodium resorption of liver cirrhosis. The administration of diuretic drugs, mainly if incorrect and/or uncontrolled, may result in a further depletion of sodium and 'effective' blood volume.

There is evidence that chronic depletion of 'effective' blood volume reduces the renal blood flow and may precipitate the renal failure (so called 'functional' renal failure: oliguria, hyponatraemia, azotaemia). In these conditions the diuretic treatment becomes less and less effective and finally dangerous.

Table 1 summarizes the main factors responsible for resistance of ascites to diuretic treatment.

TABLE 1. Factors inducing resistance to diuretic treatment

1. Hypoalbuminemia, decrease of 'effective' blood volume, hyponatraemia

2. Hypoperfusion of the kidney, abnormal distribution of intrarenal blood flow, decrease of GFR

3. Hyperaldosteronism, hyperantidiureticism

4. Renal failure

It follows that the most difficult therapeutic problem of decompensated liver cirrhosis deals with the control of diuretic-resistant ascites. A therapeutic plan has been outlined in Table 2.

TABLE 2. Treatment of resistant ascites

1. Correction of sodium depletion according to Lévy (1973)

2. Angiotensin II infusion (500-1000 $\mu \mathrm{g}$ in saline for 4-12 hr)

3. Forced saluresis (frusemide infusion: $500-1000 \mathrm{mg}$ in saline for 2-4 hr)

4. Concentration-reinfusion of ascitic fluid

Firstly, it requires restoring sodium balance which may be achieved by sodium chloride infusion according to Lévy (1973). Afterwards the abnormal distribution of intrarenal blood flow needs to be corrected: this may be achieved either by prolonged angiotensin infusion or by frusemide at high dosage. Frequently in these conditions the effects of diuretic drugs may be restored.

The intravenous infusion of ascitic fluid in the management of intractable ascites has been attempted for many years because it enables large amounts of trapped fluid to be withdrawn and infused into the effective blood circulation without protein depletion. In spite of the right pathophysiological basis, this procedure was dropped owing to the difficult technique and transient results. More recently Lévy et al. (1971) have suggested an automated device for drainage of ascitic fluid, its ultrafiltration and intra- 
venous reinjection of concentrate at a controlled rate. The overall procedure is carried out in closed circuit with disposable material for tubing and dialysis compartment (Rhodiascit).

During the past years we have evaluated the application of the concentration-reinfusion technique (CR) in twenty selected cases of liver cirrhosis with diuretic-resistant ascites. The criteria for admission of the patients to the investigation are listed in Table 3.

TABLE 3. Selection criteria of cirrhotic patients for ascites concentration-reinfusion

1. Contraindications

Heart failure, rhythm disturbances

Recent digestive bleeding

Haemorrhagic or infected ascites

2. Indications

Low serum albumin and/or total proteins

Hyponatraemia

Functional renal failure

This presentation deals with sixteen patients in which a satisfactory therapeutic effect was obtained.

In Table 4 we have summarized the fluid balance during CR sessions.

TABLE 4. Fluid balance during concentrationreinfusion sessions in sixteen cirrhotic patients with diuretic resistant ascites

\begin{tabular}{lrl}
\hline \multicolumn{1}{c}{ Parameters } & Mean & Range \\
\hline Drawn ascites (ml) & $11553^{*}$ & $7100-15800$ \\
& $20100 \dagger$ & \\
& $11600 \dagger$ & \\
Ultrafiltrate (ml) & $7893^{*}$ & $5200-11850$ \\
& $15200 \dagger$ & \\
Reinfused (ml) & $7950 \dagger$ & \\
& $3553^{*}$ & $1900-4500$ \\
Concentration (x) & $4900 \dagger$ & \\
& $3650 \dagger$ & \\
& $2 \cdot 2^{*}$ & $1 \cdot 7-3 \cdot 1$ \\
Reinfusion period (hr) & $2 \cdot 4 \dagger$ & \\
& $3 \cdot 1 \dagger$ & \\
& $13^{*}$ & $7-18$ \\
Reinfusion rate (ml/hr) & $13 \dagger^{\dagger}$ & \\
& $274^{*}$ & $250-310$ \\
& $245 \dagger$ & \\
\hline
\end{tabular}

* Mean values of fourteen patients.

$\dagger$ These values are not included in the mean and refer to two patients submitted to CR and frusemide-assisted diuresis.

The acute effects of ascites reinjection in fourteen cirrhotic patients are shown in Fig. 1. During CR session, both water and electrolyte excretion were markedly increased; there was also an increase in glomerular filtration rate (GFR) and urinary $\mathrm{Na} / \mathrm{K}$ ratio; the body weight significantly decreased.

Two examples are reported in detail. Figure 2 shows that the acute effect of $\mathrm{CR}$ on water and
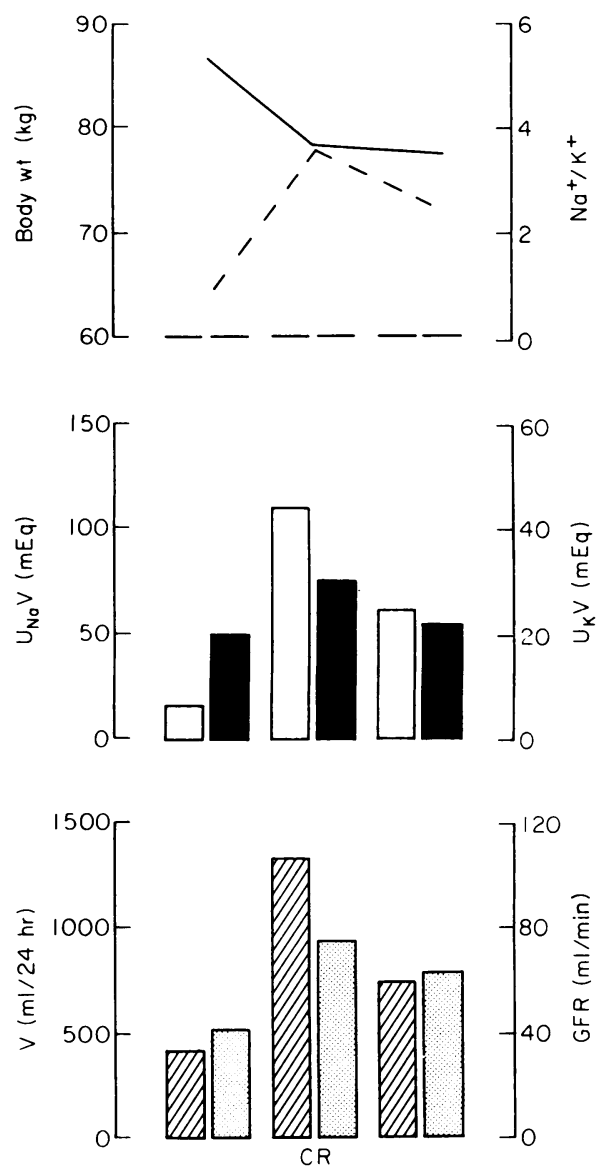

Fig. 1. Mean values for body weight (-), urinary $\mathrm{Na}^{+} / \mathrm{K}^{+}$ratio (--), daily urinary sodium excretion $\left(\mathrm{U}_{\mathrm{NaV}}\right)(\square)$, daily urinary potassium excretion $\left(\mathrm{U}_{\mathrm{K}} \mathrm{V}\right)$ $(G)$, urine volume $(\mathrm{V})(\mathbb{G})$, and glomerular filtration rate (GFR) ( $\square$ ) before, during (CR), and after the concentration-reinfusion procedure in fourteen patients.

sodium diuresis disappeared within $48 \mathrm{hr}$ from the beginning of the session in spite of a good GFR. Probably the rebound after acute water and sodium depletion plays an important role in enhancing resorption of the fluid available along the nephron. $\frac{7}{2}$ Indeed, the subsequent administration of saluretic and potassium-sparing drugs acting at different levels $\mathcal{N}$ of the renal tubule again induced diuresis and com- $N$ pleted the removal of the ascites. As shown in Fig. 3, N in some cases the duration of diuretic effect of CR was shorter than $48 \mathrm{hr}$, but again drug treatment became effective only after ascites reinjection, in $\stackrel{0}{D}$ comparison with the preinfusion period, even when $\stackrel{D}{\rightarrow}$ the same drugs were employed.

In patients with ascites and massive oedema, low

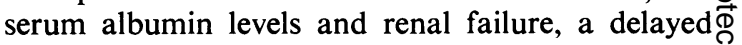
diuretic response to $\mathrm{CR}$ is almost the rule and may $\frac{\overrightarrow{\mathrm{Q}}}{\mathrm{O}}$ 


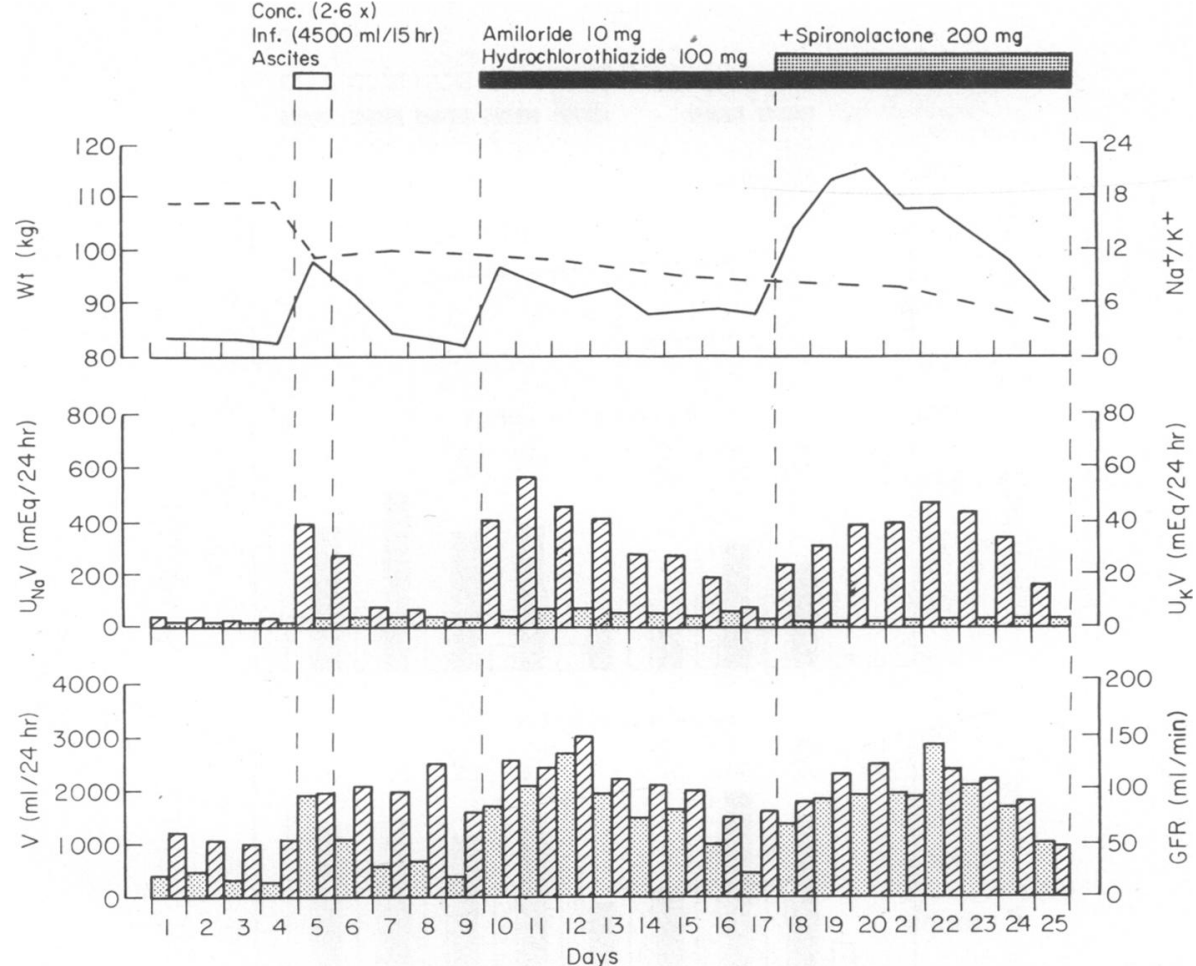

FIG. 2. Detailed studies of effect of concentration-reinfusion in one patient. Abbreviations as in Fig. 1.
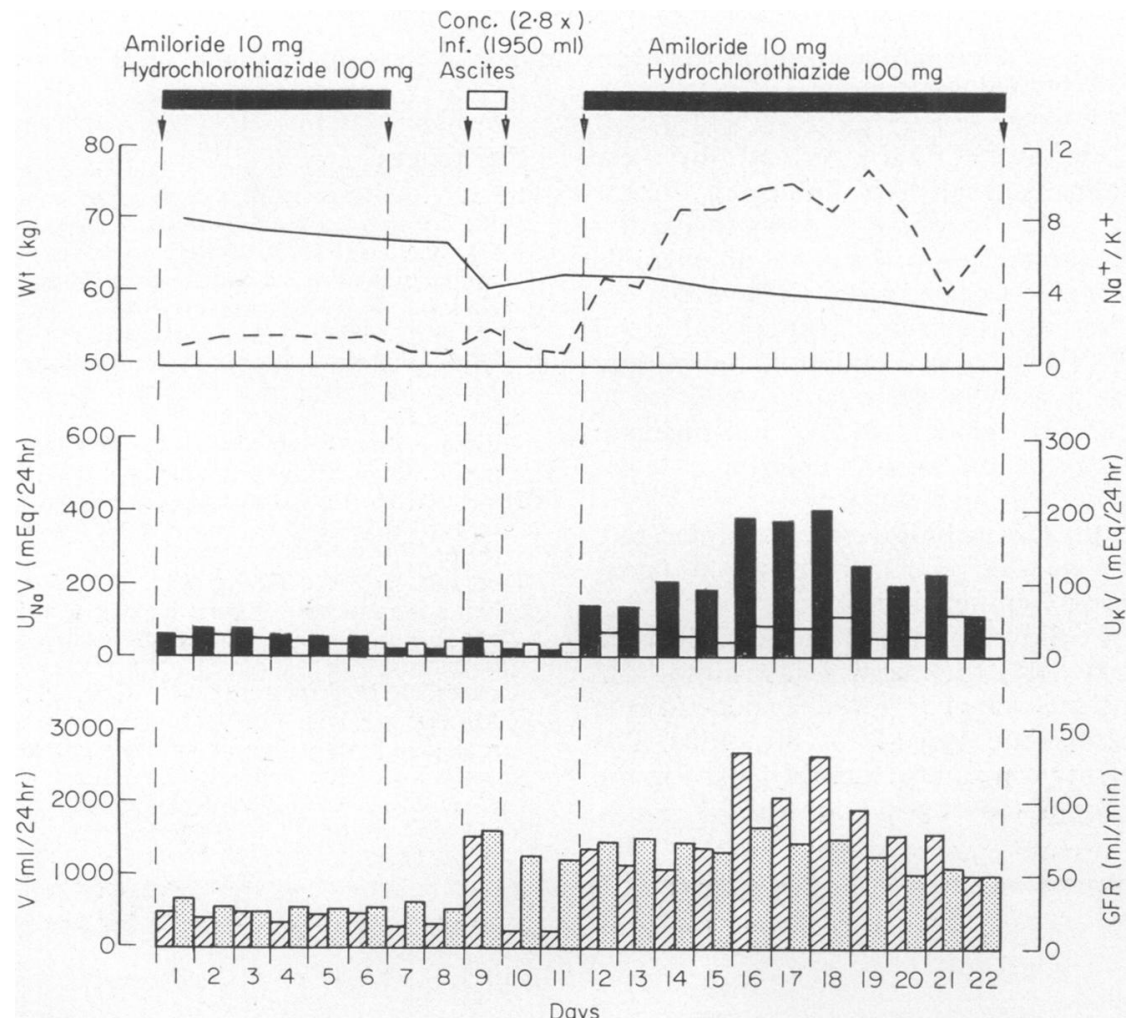

Iig. 3. Detailed studies of effect of concentration-reinfusion in one patient. Abbreviations as in Fig. 1. 

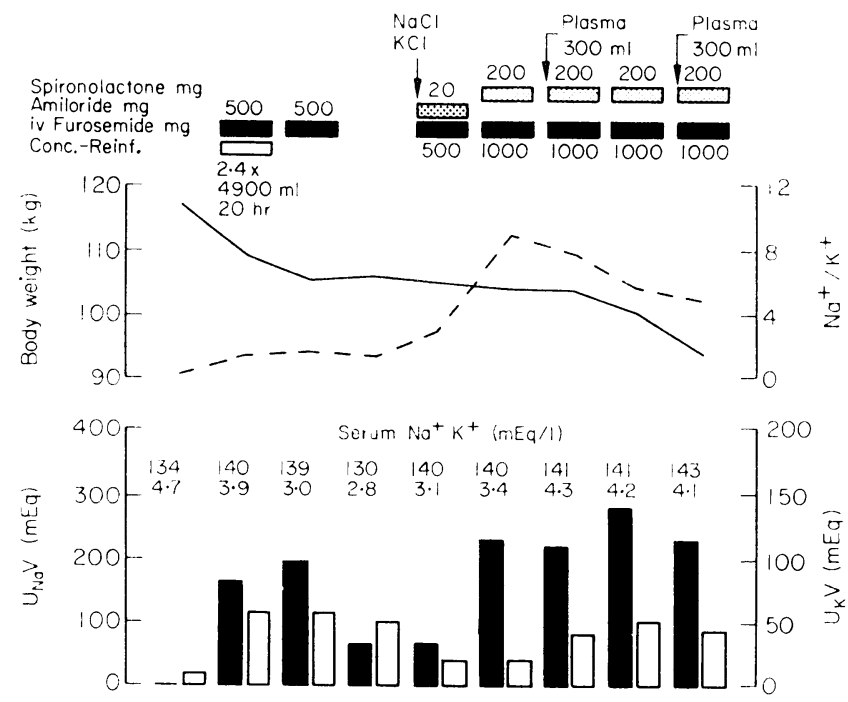

be dangerous because of fluid overload. In these cases a forced saluresis needs to be induced by means of frusemide in order to prevent possible cardiovascular complications. Figure 4 reports an example of $\mathrm{CR}$ and frusemide-assisted diuresis in a patient with tense ascites, anasarca and functional renal failure. In spite of his severe condition, the patient was submitted to controlled diuretic treatment up to complete depletion of residual ascites and oedema. Blood urea nitrogen and serum creatinine levels returned to normal during treatment.

In all patients the CR technique was well tolerated, the only consistent side effects being mild fever, transient thirst and hypotension.

As regards the follow-up, two of our patients died 7 and 10 days after CR for massive gastrointestinal bleeding. Six patients were controlled as out-patients by conventional diuretic drugs for 4-8 months. The other eight patients were unfortunately lost for the follow-up after 3 months of good control.

\section{References}

Lévy, V.G., Hecht, Y., Gilorgiacopoulos, H., Hadchouel. P., Touboul, J.P., Sausse, A., Granger, A., Stern, A. \& CArol., J. (1971) Assèchement des ascites cirrhotiques par réinjection veineuse continue du liquide d'ascite concentré. Annales de Médecine Interne, 122, 1075.

LÉvy, V.G. (1973) L'hyponatrémie des cirrhoses ascitiques. Essai dinterprétation physiopathologique. Archives francaises des Maladies de l'Appareil digestif, 62, 193.

PAPper, F. \& VAamonde, C.A. (1971) The kidney in liver disease. In: Diseases of the Kidney (Ed. by M. B. Strauss 0 and L. G. Welt), vol. I, p. 1139. Little, Brown Co., Boston.

SHERLOCK, S. (1970) Ascites formation in cirrhosis and its $\frac{D}{D}$ treatment. Scandinavian Journal of Gastroenterology, 5 을. (Suppl. 7), 9.

Tristani, F.E. \& COHN, J.N. (1967) Systemic and renal o hemodynamics in oliguric hepatic failure: effect of volume $N$ expansion. Journal of Clinical Investigation, 46, 1894.

Vlahcević, Z.R., Adham, N.F., Chalmers, T.C., Cl.hmont, R.J., MoOre, E.W., Jick, H., Curtis, G.W. \& MORRISON, R.S. (1967) Intravenous therapy of massive ascites in patients with cirrhosis. Gastrocnterologi, 53, 211. C

ViahCeVić, Z.R., Adham, N.F., Jick, H., MoOre, E.W. \& $\mathbb{D}$ Chalmers, T.C. (1965) Renal effects of acute expansion of ? plasma volume in cirrhosis. New England Journal of $\square$ Medicine, 272, 387. 